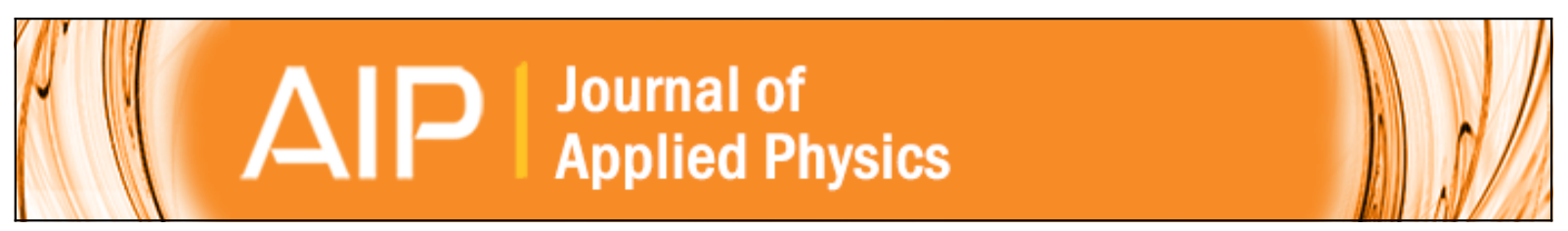

\title{
Frequency up-conversion in nonpolar a-plane GaN/AIGaN based multiple quantum wells optimized for applications with silicon solar cells
}

S. Radosavljevi, J. Radovanovi, V. Milanovi, and S. Tomi

Citation: Journal of Applied Physics 116, 033703 (2014); doi: 10.1063/1.4890029

View online: http://dx.doi.org/10.1063/1.4890029

View Table of Contents: http://scitation.aip.org/content/aip/journal/jap/116/3?ver=pdfcov

Published by the AIP Publishing

\section{Articles you may be interested in}

Optimization of InAs/AllnAs quantum wells based up-converter for silicon solar cells

J. Appl. Phys. 110, 063713 (2011); 10.1063/1.3641977

Ultrafast intersubband relaxation and nonlinear susceptibility at $1.55 \mathrm{~m}$ in GaN/AIN multiple-quantum wells Appl. Phys. Lett. 84, 1102 (2004); 10.1063/1.1647275

Well-width dependence of photoluminescence emission from a-plane GaN/AIGaN multiple quantum wells Appl. Phys. Lett. 84, 496 (2004); 10.1063/1.1638884

GaN/AIGaN multiple quantum wells on a-plane GaN pillars for stripe-geometry nonpolar ultraviolet light-emitting devices

Appl. Phys. Lett. 83, 2599 (2003); 10.1063/1.1614835

Piezoelectric field-enhanced second-order nonlinear optical susceptibilities in wurtzite GaN/AIGaN quantum wells Appl. Phys. Lett. 76, 333 (2000); 10.1063/1.125736

\section{A.P $\left.\right|_{\text {Applied Physics }} ^{\text {Journal of }}$}

Journal of Applied Physics is pleased to announce André Anders as its new Editor-in-Chief 


\title{
Frequency up-conversion in nonpolar a-plane GaN/AIGaN based multiple quantum wells optimized for applications with silicon solar cells
}

\author{
S. Radosavljević, ${ }^{1}$ J. Radovanović, ${ }^{1, a)}$ V. Milanović, ${ }^{1}$ and S. Tomić ${ }^{2}$ \\ ${ }^{1}$ School of Electrical Engineering, University of Belgrade, Bulevar kralja Aleksandra 73, 11200 Belgrade, \\ Serbia \\ ${ }^{2}$ Joule Physics Laboratory, School of Computing, Science and Engineering, University of Salford, \\ Manchester M5 4WT, United Kingdom
}

(Received 4 June 2014; accepted 1 July 2014; published online 17 July 2014)

\begin{abstract}
We have described a method for structural parameters optimization of GaN/AlGaN multiple quantum well based up-converter for silicon solar cells. It involves a systematic tuning of individual step quantum wells by use of the genetic algorithm for global optimization. In quantum well structures, the up-conversion process can be achieved by utilizing nonlinear optical effects based on intersubband transitions. Both single and double step quantum wells have been tested in order to maximize the second order susceptibility derived from the density matrix formalism. The results obtained for single step wells proved slightly better and have been further pursued to obtain a more complex design, optimized for conversion of an entire range of incident photon energies. C 2014 AIP Publishing LLC. [http://dx.doi.org/10.1063/1.4890029]
\end{abstract}

\section{INTRODUCTION}

Ongoing development of novel device concepts, materials, and fabrication processes is aimed at lowering the cost of solar power and hence at making it more competitive..$^{1-5}$ Significant research efforts are directed towards enhancing the performance of solar cells (SCs), as well as towards optimizing their fabrication and installation and improving their working efficiencies. ${ }^{6}$ The overall energy conversion capability of SCs has increased steadily in the last decade through enhanced photon absorption and charge carrier transport. The highest share in production and market belongs to solar cells based on silicon, both in crystalline and polycrystalline form due to its low price and availability, as well as the maturity of crystal growth and fabrication processes. Yet, like other single junction/single bandgap devices, they suffer from relatively weak conversion efficiency due to the wavelength mismatch between the narrow wavelength band associated with the semiconductor energy gap and the broad band of the (blackbody) emission curve of the Sun. The power loss is associated with both long-wavelength photons that do not have enough energy to excite electron-hole pairs across the energy gap (leading to a $\sim 20 \%$ loss in silicon) and shortwavelength photons that excite pairs with energy above the gap, which thereby waste the extra kinetic energy as heat.

Recently, nanoengineering approaches to enhance solar power conversion efficiency have started to receive considerable interest. ${ }^{4,5,7}$ Within such methods, sub-bandgap photons (that would normally be transmitted straight through the SC) may be utilized in a process called up-conversion (UC) to produce an above-bandgap photon, ${ }^{5,7}$ or, on the other hand, above-bandgap photons can be reduced to multiple lower energy photons in a process called down-conversion (DC). Using one or both of these processes can increase the current collected in a solar cell. One of the unique advantages of this

${ }^{\text {a)} E-m a i l: ~ r a d o v a n o v i c @ e t f . b g . a c . r s ~}$ concept is that it is compatible with many existing solar technologies, due to the fact that only the incoming solar spectrum is modified. For example, the UC layer can be attached to the rear of a bifacial solar cell without disturbing the performance of the device for the above bandgap photons. ${ }^{9}$ Consequently, any additional photocurrent that is generated via the UC results in performance enhancement. In addition, it is also possible to place a reflector behind the UC layer so that no luminescence escapes out of the rear of the device.

Semiconductor quantum well $(\mathrm{QW})$ heterostructures offers extra degree of freedom in designing the optically active transitions between quantum states in such nanostructures. By following the quantum engineering paradigm in the materials modeling, by suitable variation of semiconductor QW width, potential height of surrounding barrier material and shape of the QW itself, it is possible to tune the optically active transitions in such nanostructures to match simultaneously the wavelength of the incident light and the desired wavelength of output signal to the absorption spectra of bulk solar cell device. Moreover, by placing them in a QW array in which each of the quantum wells is with slightly different absorbing wavelengths and each of them with the exactly the same output wavelength, potentially it would be possible to achieve the broadband absorption of the sunlight with narrow output emission form such device. Another possible advantage of the up/down convertor based on the QW structures when compare to doped material is in much more suppressed detrimental Shockley-Hall-Read nonradiative recombination too. Potential advantage of QW based up (or down) converters when attached to the bulk based absorption region of the conventional solar cell, are also in the fact that they can convert the part of the sun spectra otherwise transparent (or with energy lost in the form of heath) in the single energy gap absorption region SC design without converter, for the benefit of increased efficiency of such SC design.

In order to overcome the loss of low-energy photons, we have considered a model of up-converter based on nonlinear 
optical effects related to intersubband transitions in quantum well structures. Nonlinear optical effect involves a couple of photons unusable by the solar cell, whose sum of energies is almost equal to the value of the bandgap $(E g)$ of silicon. After conversion, a high-energy photon (emitted via the transition of excited electron down to the ground state of the quantum well) can be reused by the SC. Thus, the larger portion of the solar spectrum may be exploited and the efficiency of current generation would increase.

We started by designing individual one-step QWs through varying their structural parameters (the widths of step and well layers and the $\mathrm{Al}$ content in the step region) in order to influence the positions of bound states and the shape of the corresponding wavefunctions. The goal was to optimize the light converter by searching for maximum of the relevant second order susceptibility derived from the density matrix formalism. The best solution is determined by using the generic algorithm optimization tool for selecting the optimal parameters, subject to physical and technological constraints, thus enabling us to obtain a realistic optimized quantum-well profile. In striving to benefit from larger off-diagonal matrix elements of wavefunctions of successive bound state-levels, we decided to reduce the electronic system and consider only the first three bound states to participate in the process.

A semiconductor that is to be used is such a nonlinear optical process needs to provide a large enough conduction band offset to accommodate at least three bound states. With respect to conditions listed here, we have chosen to use nonpolar a-plane nitrides ( $\mathrm{AlGaN}$ as a barrier and $\mathrm{GaN}$ as the well material), as it is the most suitable combination to our knowledge. Because of their wide direct bandgap, group III-nitrides and their alloys are the most promising materials for short-wavelength optical devices and are currently widely explored. ${ }^{10-13}$ This procedure is repeated for various combinations of incident photon energies, to cover the whole sub-bandgap part of the spectrum. These individual quantum wells should be grown in a sequence, so to form a multi-QW structure capable of converting photons "continuously." In more detail, adding a component (photon of a given energy $<E g$ ) into the spectrum is consistent with adding a corresponding $\mathrm{QW}$ to the total design. The entire multi-QW structure comprises close to 50 subsequently grown GaN/ AlGaN QWs optimized for absorbing and converting photons with different wavelengths.

\section{THEORETICAL CONSIDERATIONS}

The response of a material to the presence of electric field $(E)$ of the incident radiation can be described by polarization $(P)$

$$
P=\varepsilon_{0}\left(\chi^{(1)} E+\chi^{(2)} E^{2}+\chi^{(3)} E^{3}+\cdots\right),
$$

where $\chi$ represents the susceptibility of the material, while superscripts $i$ in $\chi^{(i)}$ refer to the order of susceptibility. In this paper, we consider the second order nonlinear susceptibility

$$
P_{i}^{(2)}\left(\omega_{p}+\omega_{q}\right)=\varepsilon_{0} \sum_{j, k} \sum_{p, q} \chi_{i j k}^{(2)} E_{j}\left(\omega_{p}\right) E_{k}\left(\omega_{q}\right),
$$

where indices $i, j, k$ are Cartesian components of the field ( $x, y, z$, subject to permutations). ${ }^{14}$ All components contribute to nonlinear polarization effects, thus summation over all frequency combinations $\omega_{p, q}$, need to be performed, while their sum is fixed $\left(\omega_{p}+\omega_{q}\right)$. Calculating the second-order contribution within the perturbation theory, where the unperturbed system is assumed not to be influenced by electric field, results in a well-known expression obtained from the density matrix formalism. ${ }^{14}$

We consider a multi-QW semiconductor structure, where the growth direction is labeled as $z$, therefore the second-order susceptibility amounts to $\chi_{z z z}^{(2)}$, since the $z$ component of the dipole matrix element is significantly larger than $x$ and $y$ components. ${ }^{15}$

In bulk semiconductor materials, as well as in semiconductor heterostructures, the scattering mechanisms exhibit considerable dependence on the wavevector and frequency. ${ }^{16-18}$ In case of "typical k" approximation this dependence in only frequency-related, while within "full k" approximation, it entails the wavevector relationship as well. ${ }^{16,17}$ In the model of exact "full k" approximation, linewidths for all the transitions have very complex correlation with both the wavevector and frequency, clearly leading to different values for different transitions. In this paper, we use the averaged values of linewidth $\gamma$, taking an equal value for all transitions, which leads to significant simplifications in the theoretical and numerical treatment of the problem considered here. We also assume that almost all electrons in the conduction band of the unperturbed system are in the ground state. All these assumptions are typically used in the literature and provide a good quantitative picture of the analyzed problem. On the other hand, a very interesting treatment for obtaining the precise values of linewidths, offered in Refs. 16-18 is worth implementing and will be considered in future work.

In addition to the above considerations, we take into account only three relevant bound states of the QW, labelled 0,1 , and 2. Starting from the general expressions detailed in Ref. 14 (Eq. (3.6.18) therein), after introducing the above assumptions, we arrive to the expression for second-order nonlinear susceptibility in the form

$$
\chi_{z z z}^{(2)}=\frac{e^{3} N}{2 \hbar^{2}} \mu_{10} \mu_{12} \mu_{02} \times Q,
$$

with

$$
\begin{aligned}
Q= & {\left[\frac{1}{\left(\omega_{10}-\omega_{p}\right)-i \gamma}+\frac{1}{\left(\omega_{10}-\omega_{q}\right)-i \gamma}\right] } \\
& \times\left[\frac{1}{\left(\omega_{20}-\omega_{p}-\omega_{q}\right)-i \gamma}+\frac{1}{\left(\omega_{21}+\omega_{p}+\omega_{q}\right)+i \gamma}\right] \\
& +\left[\frac{1}{\left(\omega_{21}+\omega_{p}\right)+i \gamma}+\frac{1}{\left(\omega_{21}+\omega_{q}\right)+i \gamma}\right] \\
& \times\left[\frac{1}{\left(\omega_{21}+\omega_{p}+\omega_{q}\right)+i \gamma}+\frac{1}{\left(\omega_{10}+\omega_{p}+\omega_{q}\right)+i \gamma}\right] .
\end{aligned}
$$


Here, $N$ is the electron number density, $\mu_{n m}=\langle n|z| m\rangle$ are the dipole matrix elements for transitions between states $n$ and $m$ of the QW (the relevant bound states are labelled 0,1 , and 2), $\omega_{p}$ and $\omega_{q}$ are the frequencies of incident photon pairs. If we consider the cases close to resonance, where $\omega_{p}=\omega_{10}+\delta$ and $\omega_{q}=\omega_{21}+\varepsilon$, with $|\delta| \ll \omega_{10}$ and $|\varepsilon| \ll \omega_{21}$, then the second summand in Eq. (4) becomes much smaller than the first one, and Eq. (3) amounts to

$$
\chi_{z z z}^{(2)}=\frac{e^{3} N}{2 \varepsilon_{0} \hbar^{2}} \mu_{10} \mu_{12} \mu_{02} \times \frac{1}{\left(\omega_{20}-\omega_{p}-\omega_{q}\right)-i \gamma}\left[\frac{1}{\left(\omega_{10}-\omega_{p}\right)-i \gamma}+\frac{1}{\left(\omega_{10}-\omega_{q}\right)-i \gamma}\right]
$$

with the term $\left[\left(\omega_{21}+\omega_{p}+\omega_{q}\right)+i \gamma\right]^{-1}$ being neglected as well. The above expression can be further simplified to

$$
\chi_{z z z}^{(2)}=\frac{e^{3} N}{2 \varepsilon_{0} \hbar^{2}} \mu_{10} \mu_{12} \mu_{02} \times \frac{1}{\varepsilon+\delta+i \gamma}\left[\frac{1}{\delta+i \gamma}+\frac{1}{\varepsilon-\Delta \omega+i \gamma}\right]
$$

where $\Delta \omega=\omega_{10}-\omega_{21}$. The absolute value of the real part of Eq. (6) is given by

$$
\left|\operatorname{Re}\left\{\chi_{z z z}^{(2)}\right\}\right|=\frac{e^{3} N}{2 \varepsilon_{0} \hbar^{2}}\left|\mu_{02} \mu_{21} \mu_{10}\right| \frac{\delta(\Delta \omega-\varepsilon)(\delta+\varepsilon)(\delta+\varepsilon-\Delta \omega)+\gamma^{2}\left[2 \gamma^{2}+(\Delta \omega-\varepsilon)(\Delta \omega+\delta)-\varepsilon \delta\right]}{\left[(\varepsilon+\delta)^{2}+\gamma^{2}\right]\left(\delta^{2}+\gamma^{2}\right)\left[(\varepsilon-\Delta \omega)^{2}+\gamma^{2}\right]} .
$$

On the other hand, if we take a strictly resonant case $\left(\omega_{p}=\omega_{10}, \delta=0\right)$, then Eq. (7) reduces to

$$
\left|\operatorname{Re}\left\{\chi_{z z z}^{(2)}\right\}\right|=\frac{e^{3} N}{\varepsilon_{0} \hbar^{2}}\left|\mu_{10} \mu_{12} \mu_{02}\right| \frac{1+\frac{\Delta \omega(\Delta \omega-\varepsilon)}{2 \gamma^{2}}}{\left(\varepsilon^{2}+\gamma^{2}\right)\left(1+\frac{(\Delta \omega-\varepsilon)^{2}}{\gamma^{2}}\right)} .
$$

Our goal is to optimize the real part of the susceptibility in order to enhance the response of the material at frequency $E_{g} / \hbar$ (with $E_{g}$ being the energy bandgap of $\mathrm{Si}$ ) which is best absorbed by the silicon solar cell. Hence we start by setting $\omega_{p}+\omega_{q}=E_{g} / \hbar$ in Eq. (5). In order to successfully use the Genetic Algorithm (GA) for global optimization, ${ }^{19-21}$ as the tool for varying the QW parameters, we must formulate a suitable target function. GA is inspired by Darvin's theory of evolution, i.e., it is modeled according to the idea of selecting the fittest individuals in a current iteration of the algorithm and forwarding them to the next cycle to be used to create a new set of solutions, while less successful individuals are dismissed. Starting from an initial set of solutions to the problem, analogous to survival of superior individuals in nature through generations, after a large number of iterations, GA returns the best solution within the search space that meets the predefined criteria. A current set of solutions which are considered during one iteration is called the population. The initial population can be either randomly generated by the algorithm or preset, if there are indications where the optimum of the defined fitness (target) function should be searched for. The solutions from one population are used to form a new population (offsprings) which is expected to show progress compared to the individuals from previous population, so called "parents." Current parents are selected according to their fitness (target function value) - the more suitable they are, the more chances they have to reproduce, which is achieved by processes of crossover, mutation, and elitism. Crossover produces new individuals by directly recombining two parents to form an offspring, while mutations introduce certain changes in defining parameters of a promising parent. These changes are necessary in order to avoid converging towards local optima. In order to preserve the parents that are more suitable than offsprings in the current population, that would otherwise be lost after crossover and mutation, the term of elitism is brought in. One or more parents with top scores are considered elite members and are automatically transported to the next generation. This evolution-like iterative process is repeated until some condition (for example, the total number of populations or improvement of the best solution) is satisfied.

GA is usually implemented to process functions of the type $f\left(x_{1}, x_{2} \ldots x_{i} \ldots x_{n}\right)$, which depend on an arbitrary number of independent variables: $x_{1}, x_{2} \ldots x_{i} \ldots x_{n}$. In this paper, GA is used to find the best solution for the QW profile in terms of maximizing the second order susceptibility given by Eq. (6) (more precisely, the absolute value of its real part), so the fitness function may be defined as

$$
F=\frac{\left|\operatorname{Re}\left\{\chi_{z z z}^{(2)}\right\}\right|}{N e / \varepsilon_{0}}
$$

where $\left|\operatorname{Re}\left\{\chi_{z z z}^{(2)}\right\}\right|$ is given by Eq. (8).

\section{RESULTS AND DISCUSSION}

To find the optimized multi-QW structure, we have started from individual step QWs where the well material is 
taken as pure nonpolar a-plane $\mathrm{GaN}$, the barrier material is pure AlN (to provide a high enough potential barrier), while the step layer comprises $\mathrm{Al}_{x} \mathrm{Ga}_{1-x} \mathrm{~N}$. For calculating the electronic states, we used a one band model that takes into consideration the effects of strain and band nonparabolicity. ${ }^{22,23}$ In one-step quantum wells, the following input arguments are needed for evaluating the second order susceptibility: the widths of step and well layers and the content of AlN in the step regions. GA algorithm needs to be supplied with realistic upper and lower boundaries for these parameters. In numerical calculations, we have used the following values of additional parameters: $m_{\text {GaN }}=0.209 m_{0}$, $m_{A I N}=0.329 m_{0}$ (where $m_{0}$ is the free electron mass), ${ }^{10}$ $E_{g A I N}=6.47 \mathrm{eV}, E_{g G a N}=3.24 \mathrm{eV},{ }^{11}$ and the conduction to valence band offset ratio is taken as 60:40. ${ }^{12}$ The influence of strain is included by using parameters from Ref. 10, so the outer barrier height amounts to $U_{b}=1.76 \mathrm{eV}$. The relaxation parameters in Eq. (5) are considered to be equal for all transitions and set to $5 \mathrm{meV}$ which is a typical value used in the literature, and the energy gap of $\mathrm{Si}$ is taken as $1.12 \mathrm{eV}$.

The first set of calculations was performed on one-step QWs, by using the genetic algorithm to find the best possible value of the fitness function (9), regardless of the individual photon energies $\omega_{p}, \omega_{q}$ but fixing $\hbar\left(\omega_{p}+\omega_{q}\right)=1.12 \mathrm{eV}$. The optimal QW profile obtained by this procedure has the total width equal to $2.27 \mathrm{~nm}$, with the $1.22 \mathrm{~nm}$ wide step, and with step height of $U_{s}=0.483 \mathrm{eV}$, as presented in Fig. 1 . These parameters yield $F=1.35 \times 10^{-18} \mathrm{~cm}^{3} / \mathrm{V}^{2}$, or $\left|\chi_{z z z}^{(2)}\right|$ $=1.35 \times 10^{-18} \mathrm{~cm}^{3} / \mathrm{V}^{2} \times\left(e N / \varepsilon_{0}\right)$.

In order to try to improve this result, the next run of the GA algorithm is performed for a modified quantum well encompassing two internal step layers. The extra potential step introduces two additional free parameters for optimization (its width and height), which should provide more freedom in shaping the optimal profile. However, this did not lead to an increased values of the fitness function, and the reason is in the large energy separation between the ground and the second excited state $(\sim 1.12 \mathrm{eV})$ required. Apparently, such separation can only be achieved in deep and narrow QWs and the insertion of additional step layer increases the well width (the minimal width of the second layer is limited to at least $0.8 \mathrm{~nm}$ to remain realizable). Fig. 2

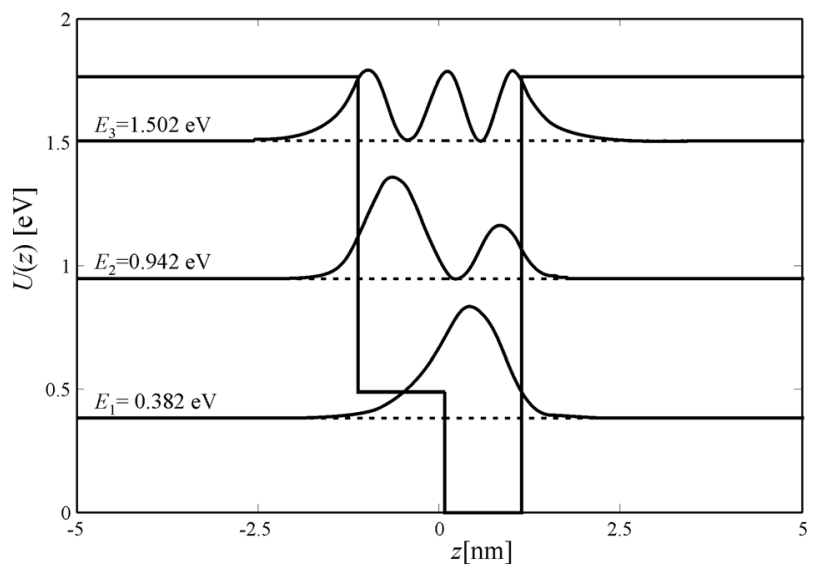

FIG. 1. The conduction band profile of the optimized single-step quantum well up-converter for Si solar cell.

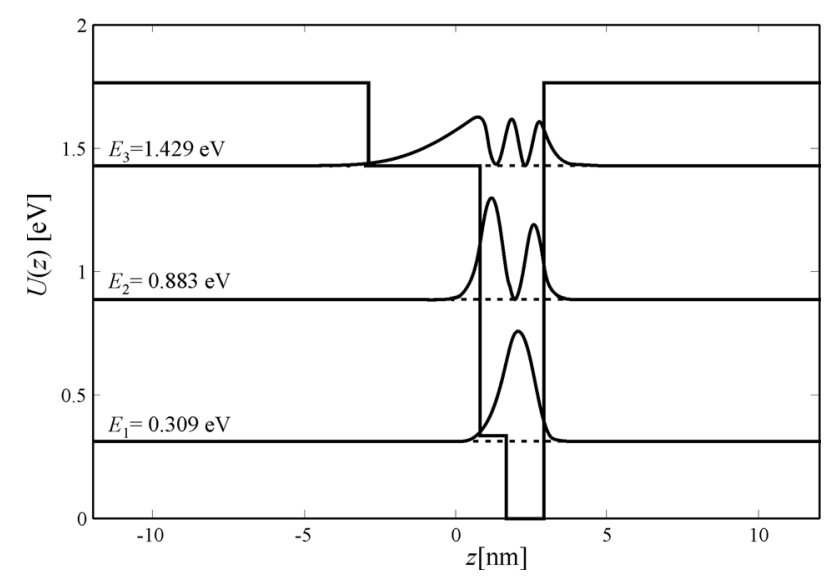

FIG. 2. The optimized double-step quantum well profile.

illustrates the profile of the optimized two-step QW comprising the following step-layers: one layer is $3.69 \mathrm{~nm}$ wide with the $\mathrm{Al}$ mole fraction of $x_{S 1}=0.81$ and the other is $0.9 \mathrm{~nm}$ wide with $x_{S 2}=0.19$, while the total well width is $5.81 \mathrm{~nm}$. The corresponding value of the second order susceptibility (scaled by the $\left(e N / \varepsilon_{0}\right)$ constant) is $0.52 \times 10^{-18} \mathrm{~cm}^{3} / \mathrm{V}^{2}$, which is clearly below the one-step QW result.

Having determined that the introduction of an additional step layer yields no improvement in the fitness function, we have returned to the one-step QW optimization, repeating the procedure for the almost complete sub-bandgap energy range with $10 \mathrm{meV}$ increments in energy. In other words, the energy of one incident photon is varied in the $\left(E_{g S i} / 2, E_{\max }\right)$ range, where $E_{\max }=1.06 \mathrm{eV}$ is the closest we could get to $E_{g S i}$ and still obtain meaningful results. The energy of the second photon is thus fixed at $E_{g S i}-\hbar \omega$, causing the symmetry of the plot presented in Fig. 3, i.e., all the energies from the range $\left[E_{g S i}-E_{\max }, E_{\max }\right]$ are encompassed. For each energy value $\hbar \omega$, we have performed the QW profile optimization with respect to the fitness function (9). Individual QWs obtained by this procedure should form a sequence

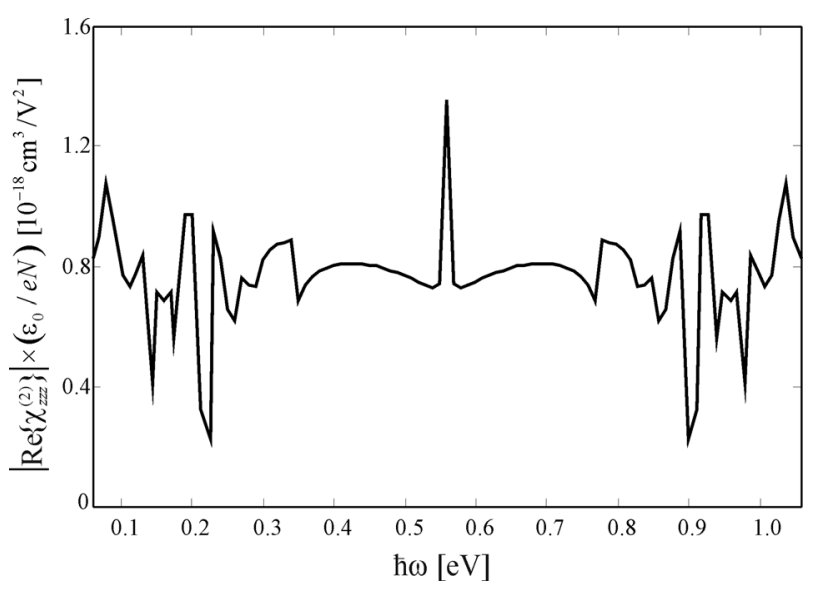

FIG. 3. The scaled value of the real part of the second order nonlinear optical susceptibility as a function of the incident photon energy. Each point in this figure refers to the optimal result obtained for an individual quantum well within the multi-QW converter, optimized for a particular value of the incident photon energy (the energy of the second photon is evidently $\left.E_{g S i}-\hbar \omega\right)$. 


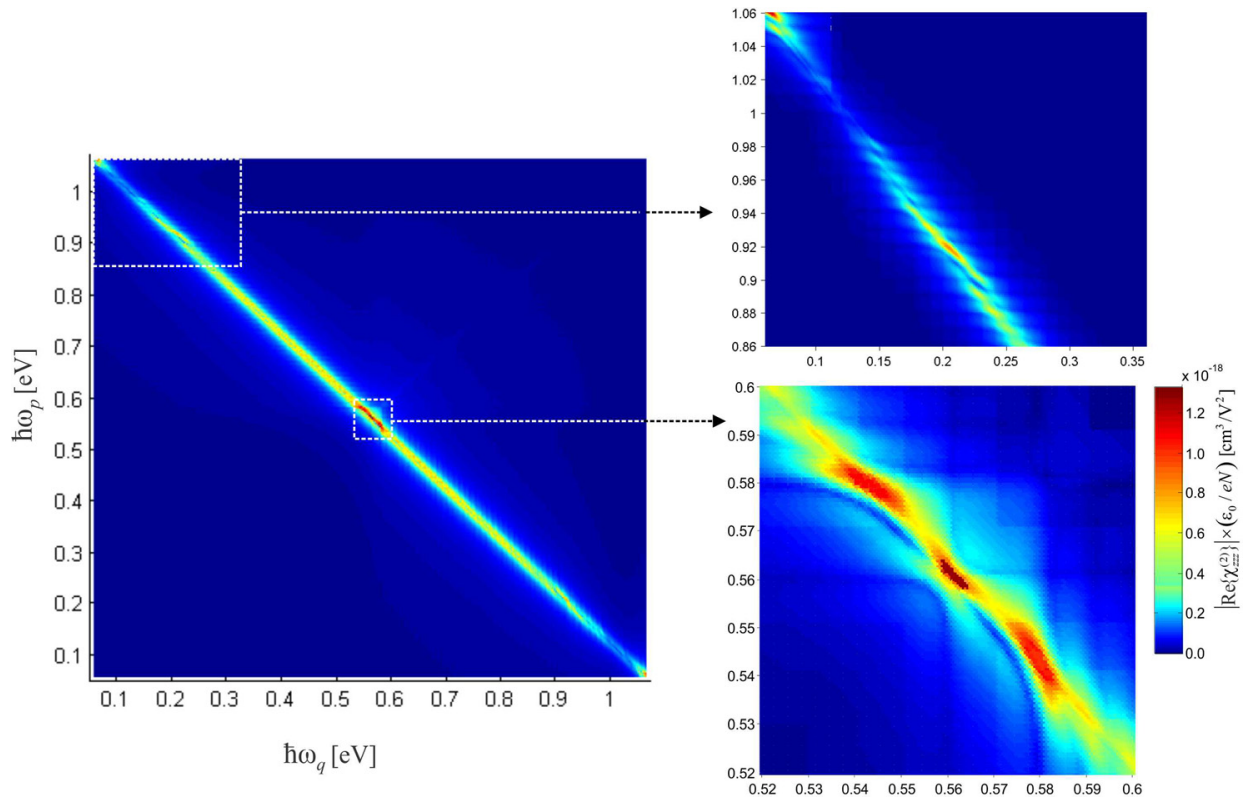

FIG. 4. The scaled values of the real part of the second order nonlinear optical susceptibility in a more general, non-resonant case, as a function of incident photon energies.

(i.e., a multi-QW structure) capable of performing an almost continuous energy conversion of incoming radiation.

We observe that the parameters of individual QWs (such as step and well layer widths and the Al content in the step regions) can be tuned with high precision for a pair of photon energies. Photons with frequencies at the end of the spectrum determined by the silicon bandgap are more difficult to accommodate as explained above. Furthermore, there is a narrow range of photon energies near the center $\left(E_{g S i} / 2\right)$ where the fitness function drops rapidly (Fig. 3), due to specific requirements for the QW profile that apparently cannot be best achieved with the chosen set of free parameters and the material system in question. One possibility is to allow for the variations of the outer barriers composition (AlGaN with high $\mathrm{Al}$ content rather than pure $\mathrm{AlN}$ ), which may lead to an improvement of the target function, but complicate the overall multi-QW design.

Finally, we have performed a set of calculations for the optimized sequence of QWs, taking into account nearly resonant photons according to Eq. (7), and varying the incident photon energies $\hbar \omega_{p}$ and $\hbar \omega_{q}$. The obtained results are presented in Fig. 4.

It is clear from the above figure, that only the points near the diagonal (very close to resonant conditions, with $\varepsilon, \delta \rightarrow 0$ ) have significant values, and the susceptibility drops rapidly as the sum of $\hbar \omega_{p}$ and $\hbar \omega_{q}$ departs from $E_{g}$ of silicon, for which the converter structure is constructed. The brown/red areas of Fig. 4 correspond to the highest values of the real part of susceptibility.

\section{CONCLUSION}

Silicon is nowadays the most commercially used material for manufacturing solar cells; however, its energy gap significantly limits the spectrum of daylight that can be directly absorbed. Typical loss mechanisms include the transmission of sub-bandgap light and the thermalization of charge carriers generated by the absorption of photons with energies greater than the bandgap. Up-conversion of low energy photons is a promising approach to reduce this portion of the losses, as an up-converter generates one highenergy photon out of two low-energy photons, which then creates free charge carriers in the solar cell. This was the focus of our paper, and the described procedure based on the use of genetic algorithm global optimization tool, allowed us to vary the structural parameters of nonpolar-nitride quantum wells in search of the best step QW profile, offering highest value of the relevant nonlinear susceptibility. The method was extended to obtain a multi quantum well up-converter structure which comprises a sequence of about $50 \mathrm{GaN} /$ AlGaN QWs, each optimized for conversion of photons with particular sub-bandgap energies. The a-plane non-polar nitride material system proved suitable for providing a large enough conduction band offset to accommodate three bound states with sufficient energy separation.

\section{ACKNOWLEDGMENTS}

This work was supported by the Ministry of Education, Science and Technological Development (Republic of Serbia), ev. no. III 45010 and NATO SfP Grant, ref. no. ISEG.EAP.SFPP 984068. S.T. wish to thank the Royal Society, London, grant "High Performance Computing in Modelling of Innovative Photo-Voltaic Devices" and Great Britain Sasakawa Foundation. We would like to thank Urs Aeberhard for useful discussions and suggestions.

\footnotetext{
${ }^{1}$ M. Green, Prog. Photovoltaics 9, 123 (2001).

${ }^{2}$ R. R. King, Nat. Photonics 2, 284 (2008).

${ }^{3}$ S. Tomić, T. S. Jones, and N. M. Harrison, Appl. Phys. Lett. 93, 263105 (2008).

${ }^{4}$ T. Trupke, M. Green, and P. Wurfel, J. Appl. Phys. 92, 1668 (2002).

${ }^{5}$ T. Trupke, M. Green, and P. Wurfel, J. Appl. Phys. 92, 4117 (2002).

${ }^{6}$ W. Shockley and H. J. Queisser, J. Appl. Phys. 32, 510 (1961).

${ }^{7}$ N. Prodanović, J. Radovanović, V. Milanović, and S. Tomić, J. Appl. Phys. 110, 063713 (2011)

${ }^{8}$ Y. Yi, L. Zhu, and Z. Shuai, Macromol. Theory Simul. 17, 12 (2008).

${ }^{9}$ S. Fischer, J. C. Goldschmidt, P. Löper, G. H. Bauer, R. Brüggemann, K. Krämer, D. Biner, M. Hermle, and S. W. Glunz, J. Appl. Phys. 108, 044912 (2010).
} 
${ }^{10}$ S. Schulz, T. J. Badcock, M. A. Moram, P. Dawson, M. J. Kappers, C. J. Humphreys, and E. P. O'Reilly, Phys. Rev. B 82, 125318 (2010).

${ }^{11}$ P. Rinke, M. Winkelnkemper, A. Qteish, D. Bimberg, J. Neugebauer, and M. Scheffler, Phys. Rev. B 77, 075202 (2008).

${ }^{12}$ T. J. Badcock, P. Dawson, M. J. Kappers, C. McAleese, J. L. Hollander, C. F. Johnston, D. V. Sridhara Rao, A. M. Sanchez, and C. J. Humphreys, J. Appl. Phys. 105, 123112 (2009).

${ }^{13}$ S. Tomić and N. Vukmirović, Phys. Rev. B 79, 245330 (2009).

${ }^{14}$ R. V. Boyd, Nonlinear Optics (Academic Press-Elsevier Science, 2003), pp. $129-176$.
${ }^{15}$ V. Milanović, Z. Ikonić, and D. Tjapkin, Phys. Low-Dimens. Struct. 7, 65 (1995).

${ }^{16}$ T. Schmielau and M. Pereira, Phys. Status Solidi B 246, 329 (2009).

${ }^{17}$ T. Schmielau and M. Pereira, Microelectron. J. 40, 869 (2009).

${ }^{18}$ T. Schmielau and M. Pereira, Appl. Phys. Lett. 95, 231111 (2009).

${ }^{19}$ G. Goldoni and F. Rossi, Opt. Lett. 25, 1025 (2000).

${ }^{20}$ A. Franceschetti and A. Zunger, Nature 402, 60 (1999).

${ }^{21}$ J. Radovanović, V. Milanović, Z. Ikonić, and D. Indjin, J. Appl. Phys. 99, 73905 (2006)

${ }^{22}$ S. Tomić, A. G. Sunderland, and I. J. Bush, J. Mater. Chem. 16, 1963 (2006).

${ }^{23}$ C. Sirtori, F. Capasso, I. Faist, and S. Scandolo, Phys. Rev. B 50, 8663 (1994). 